Research Paper

\title{
Long-term results of Gamma Knife Radiosurgery for Postsurgical residual or recurrent nonfunctioning Pituitary Adenomas
}

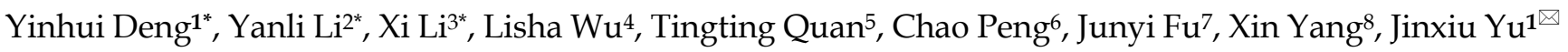 \\ 1. Department of Radiotherapy, The Second Affiliated Hospital of Guangzhou Medical University, Guangzhou, Guangdong, China. 510260. \\ 2. Department of Endocrinology, The Second Affiliated Hospital of Guangzhou Medical University, Guangzhou, Guangdong, China. 510260. \\ 3. Department of Radiology, The Second Affiliated Hospital of Guangzhou Medical University, Guangzhou, Guangdong, China. 510260. \\ 4. Department of Medical Oncology, Sun Yat-sen Memorial Hospital, Sun Yat-sen University, Guangzhou, China. 510120. \\ 5. Department of Radiology, Sun Yat-sen University Cancer Center, State Key Laboratory of Oncology in South China, Collaborative Innovation Center for \\ Cancer Medicine, Guangzhou, Guangdong, China, 510060. \\ 6. Department of Neurosurgery, Guangdong Provincial People's Hospital, Guangdong Academy of Medical Sciences, Guangzhou, Guangdong, China. \\ 510080. \\ 7. Department of Neurology, The Second Affiliated Hospital of Guangzhou Medical University, Guangzhou, Guangdong, China. 510260. \\ 8. Department of Thoracic Surgery, The Second Affiliated Hospital of Guangzhou Medical University, Guangzhou, Guangdong, China. 510260. \\ *These authors contributed equally to this work. \\ $\triangle$ Corresponding author: Jinxiu Yu at Department of Radiotherapy, The Second Affiliated Hospital of Guangzhou Medical University, Guangzhou, \\ Guangdong, China. 510260. E-mail: josse_yu@foxmail.com.
}

(C) The author(s). This is an open access article distributed under the terms of the Creative Commons Attribution License (https://creativecommons.org/licenses/by/4.0/). See http://ivyspring.com/terms for full terms and conditions.

Received: 2020.04.18; Accepted: 2020.05.21; Published: 2020.06.18

\begin{abstract}
Introduction: The aim of this retrospective study was to analyze the long-term outcomes and factors associated with treatment failure of Gamma Knife radiosurgery (GKRS) for postsurgical residual or recurrent nonfunctioning pituitary adenomas (NFPAs).

Design and Methods: $A$ total of 148 cases of postsurgical residual or recurrent NFPA patients were enrolled in the study. There were 111 cases with residual tumor and 37 cases with recurrent tumor. The median age was 46.0 years (Range: 10.9-75.8 years). The median tumor volume at GKRS was $3.6 \mathrm{~cm}^{3}$ (Range: 0.3-74.5 $\mathrm{cm}^{3}$ ), and the median tumor margin dose was $14.0 \mathrm{~Gy}$ (Range: 9 - $20 \mathrm{~Gy}$ ).

Results: Tumor shrunk in 111 patients (75\%), remained stable in 17 patients $(11.5 \%)$, and progressed in 20 patients (13.5\%) during a median of 64.5 months (Range: 14.5 - 236.0 months) of imaging follow-up. The progression-free survival rates were $99 \%, 91 \%, 88 \%$ and $74 \%$ at $1,3,5$ and 10 years after GKRS, respectively. In a multivariate analysis, tumor margin dose $(<13 \mathrm{~Gy})$ was significantly associated with tumor progression (hazard ratio $=3.526,95 \%$ confidence interval $=1.400-8.877, p=0.007$ ). New hypopituitarism occurred in 22 out of 80 patients $(27.5 \%)$, including hypogonadism $(n=7)$, hypothyroidism $(n=9)$, hypocortisolism $(n=15)$ and growth hormone deficiency $(n=1)$. In univariate and multivariate analysis, there were no factors significantly associated with new hypopituitarism. Six patients $(4.1 \%)$ developed new or worsening visual dysfunction. Four patients (2.7\%) developed new cranial neuropathy.

Conclusion: In this study, GKRS can offer a high tumor control rate as well as a low rate of complications in postsurgical residual or recurrent NFPA patients.
\end{abstract}

Key words: Gamma Knife; radiosurgery; nonfunctioning; pituitary adenoma

\section{Introduction}

Nonfunctioning pituitary adenomas (NFPAs) do not secrete biologically active hormones and represent almost $30 \%$ of pituitary tumors [1]. Usually, NFPAs may be detected because of clinical signs and symptoms such as headache, visual impairment and hypopituitarism, which are attributed to tumor 
compression. Transsphenoidal resection with minimal postoperative complications is widely recommended as the first-line treatment for NFPAs, especially for large tumors. Nevertheless, the incidence of recurrent tumor was reported to be 10\%-20\% following gross-total transsphenoidal resection for nonfunctioning macroadenomas [2-6]. The tumor progression following subtotal resection without adjuvant therapy ranged from $50 \%$ to $60 \%$ [2-5]. Under this circumstance, further management is generally required for residual or recurrent NFPA patients after surgical resection. GKRS has been applied in the treatment of NFPAs for decades which can provide a 10-year tumor control rate of $83 \%-92 \%$ as well as a low rate of hypopituitarism, varying from $9 \%$ to $32 \%$ [7-15]. There are more than 26 years of experience using Gamma Knife (Elekta, Stockholm, Sweden) at the Second Affiliated Hospital of Guangzhou Medical University since 1993. To investigate the long-term outcomes and factors associated with treatment failure of GKRS for postsurgical residual or recurrent NFPA patients, we performed a single-center retrospective study.

\section{Methods}

\section{Patient population}

Between December 1993 and December 2016, one hundred and forty-eight cases of postsurgical residual or recurrent NFPA patients underwent GKRS at the Second Affiliated Hospital of Guangzhou Medical University were enrolled in this retrospective study. All patients had at least 12 months of followup. This retrospective study was approved by the institutional committee of the Second Affiliated Hospital of Guangzhou Medical University.

\section{Clinical and radiological evaluations}

All patients were followed up with magnetic resonance (MR) imaging of the sellar, clinical and endocrinological evaluations. The follow-up evaluations were reviewed by clinicians and radiologists.

Tumor dimensions were obtained manually from MR imaging in the axial, sagittal, and coronal planes. The dimensional indices of the tumors were measured and recorded in 3 orthogonal planes: transverse (TR), anteroposterior (AP), and craniocaudal (CC). The tumor volumes were estimated using the following formula: $V=(\Pi \times[T R \times$ $\mathrm{AP} \times \mathrm{CC}]) / 6$ [16]. Unfortunately, all of the digital data from model B Leksell Gamma Knife (from 1993 to 2014) in Gamma plan was missing. So, tumor volumes were calculated based on the measurement of tumor diameters. Taking into account the irregular shape of postsurgical residual or recurrent pituitary adenomas, tumor volume measurement was only a rough estimate of the actual volume. Tumor progression was defined as tumor enlargement at least $20 \%$ in tumor volume or tumor regrowth. Tumor shrinkage was defined by at least $20 \%$ shrinkage in tumor volume. Stable tumor was defined as tumor volume change within $20 \%$. Parasellar invasion was defined as the Knosp grade 3 or 4 . Suprasellar extension was defined as the tumor close to the optic nerve and chiasm $(<2$ $\mathrm{mm})$.

Endocrinological evaluations included measurements of cortisol, adrenocorticotropic hormone $(\mathrm{ACTH})$, prolactin (PRL), growth hormone $(\mathrm{GH})$, insulin-like growth factor-1 (IGF-1), thyrotropin-stimulating hormone (TSH), free triiodothyronine (FT3), free thyroxine (FT4), luteinizing hormone (LH), follicle-stimulating hormone $(\mathrm{FSH})$, testosterone in men and estradiol levels in premenopausal women. New hypopituitarism was defined by requiring hormone replacement or a new deficiency in any one of the hormonal axes after GKRS. TSH deficiency was based on low FT4 (Normal Range: 12.00-22.00 pmol/L) with a low, normal or mildly elevated TSH. ACTH deficiency was defined as morning cortisol (08:00) level was $<100 \mathrm{nmol} / \mathrm{L}$. If morning cortisol level was $>400 \mathrm{nmol} / \mathrm{L}, \mathrm{ACTH}$ deficiency was excluded. If morning cortisol $\geq 100 \mathrm{nmol} / \mathrm{L}$ and $<400 \mathrm{nmol} / \mathrm{L}$, a stimulation test was used to confirm ACTH deficiency. Gonadotrophin deficiency in males was defined by low serum testosterone levels (Normal Range: $0.18-0.78 \mathrm{nmol} / \mathrm{L}$ ) without elevated $\mathrm{LH}$ and FSH levels. Gonadotrophin deficiency in females was defined by amenorrhea with low serum estradiol and low gonadotrophins in premenopausal females, and the absence of high gonadotrophins (LH and FSH) in menopausal women. GH deficiency was based on a low age- and sex-adjusted IGF-1 or an insufficient rise in $\mathrm{GH}$ levels during a stimulation test. As many patients were from long distances and had poor compliance, some patients did not routinely follow up with endocrine evaluation and dynamic testing.

\section{Gamma knife radiosurgery technique}

Model B Leksell Gamma Knife Unit was performed from 1993 to 2014 and Perfexion Unit (Elekta Instrument, Inc.) thereafter. Thin-slice stereotactic MR imaging with contrast was performed with axial and coronal images through the sella following stereotactic Leksell frame placement. Subsequently, the planning of GKRS treatment was made in consultation with a medical physicist, radiation oncologist and neurosurgeon. The prescribed dose was adjusted depending on tumor volume, distance to the optic nerve and chiasm, and 
history of previous radiotherapy. The maximal dose to the optic nerve and chiasm was restricted to $9 \mathrm{~Gy}$, and to the lateral wall of the cavernous sinus was restricted to 15 Gy. Small collimators of $4 \mathrm{~mm}$ and 8 $\mathrm{mm}$ were mainly adopted to achieve better GKRS treatment plan conformality.

\section{Statistical analysis}

The prognostic value of different variables relative to tumor control and new hypopituitarism were confirmed by univariate and multivariate analysis. Log-rank test statistics and a step forward likelihood ratio method of Cox proportional hazard models were applied for univariate analysis and multivariate analysis respectively. Kaplan-Meier curves were plotted for progression-free survival (PFS) and new hypopituitarism. Probability values $<0.05$ were defined as statistically significant. For statistical analysis, IBM's SPSS (version 21.0) was used.

\section{Results}

\section{Patient characteristics}

One hundred and forty-eight patients who had undergone GKRS for postsurgical residual or recurrent NFPAs between December 1993 and December 2016 were selected from the single-center. The patient population consisted of 80 male (54.1\%) and 68 female $(45.9 \%)$ patients with a median age of 46.0 years (Range: 10.9-75.8 years). The median follow-up was 64.5 months (Range: 14.5-236.0 months). There were 111 patients $(75 \%)$ with residual tumor after surgery and 37 patients (25) with recurrent tumor. The median time between first surgery and GKRS was 6.0 months (Range: 0.9 - 326.7 months). The median tumor volume at GKRS was 3.6 $\mathrm{cm}^{3}$ (Range: $0.3-74.5 \mathrm{~cm}^{3}$ ). There were 140 patients $(94.6 \%)$ with suprasellar extension and 77 patients (52.0\%) with parasellar invasion before surgical resection. Only one patient had previous radiotherapy. Ninety-four patients $(63.5 \%)$ had visual dysfunction. There are 15 patients $(10.1 \%)$ who presented with cranial nerve $(\mathrm{CN})$ dysfunction before GKRS (Table 1).

The median tumor margin dose was $14 \mathrm{~Gy}$ (range, 9-20 Gy) at a median prescription isodose $40 \%$ (Range: $30-71 \%$ ). The median maximum dose was 33 Gy (Range: 14-50 Gy) (Table 1).

\section{Tumor control}

Tumor control was confirmed by follow-up MR imaging in 128 patients (86.5\%). The data was shown in Table 2. Tumor shrank in 111 patients $(75 \%)$ and remained stable in 17 patients $(11.5 \%)$. Tumor progression was confirmed in 20 patients $(13.5 \%)$
(Figure 1). The median time of tumor progression was 35.1 months (Range: 10.7 - 99.2 months) after GKRS. Of the 16 patients with progression due to tumor regrowth, 9 patients underwent repeat GKRS, 2 patients underwent surgery, 2 patients underwent close observation, and 3 patients were lost to follow-up. Of the 4 patients with progressive cystic enlargement, 2 patients underwent surgery, 2 patients underwent close observation. The progression-free survival rates were $99 \%, 91 \%, 88 \%$ and $74 \%$ at $1,3,5$ and 10 years after postsurgical GKRS respectively (Figure 2).

Table 1. Characteristics of 148 patients with postsurgical residual or recurrent nonfunctioning pituitary adenomas and GKRS parameters

\begin{tabular}{|c|c|}
\hline Characteristic & Value \\
\hline Male/Female, n (\%) & $80 / 68(54.1 / 45.9)$ \\
\hline Median age, (range), years & $46.0(10.9-75.8)$ \\
\hline Median FU length, (range), months & $64.5(14.5-236.0)$ \\
\hline Prior surgery, once, $\mathrm{n}(\%)$ & $125(84.5)$ \\
\hline Twice, n (\%) & $22(14.9)$ \\
\hline Three times, n (\%) & $1(0.7)$ \\
\hline \multicolumn{2}{|l|}{ Tumor type, n (\%) } \\
\hline Residual tumor & $111(75)$ \\
\hline Recurrent tumor & $37(25)$ \\
\hline Months between 1st surgery and GKRS, median (range) & $6.01(0.9-326.7)$ \\
\hline Median tumor volume at GKRS, (range), $\mathrm{cm}^{3}$ & $3.6(0.3-74.5)$ \\
\hline Parasellar invasion, $\mathrm{n}(\%)$ & $77(52.0)$ \\
\hline Suprasellar extension, $\mathrm{n}(\%)$ & $140(94.6)$ \\
\hline Previous radiotherapy, n (\%) & $1(0.7)$ \\
\hline \multicolumn{2}{|l|}{ Visual function before GKRS, n (\%) } \\
\hline Visual field defect and/or visual acuity decrease & $94(63.5)$ \\
\hline Normal & $54(36.5)$ \\
\hline Pre-GKRS cranial nerve dysfunction, n (\%) & $15(10.1)$ \\
\hline \multicolumn{2}{|l|}{ GKRS parameters } \\
\hline Median tumor margin radiation dose, (range), Gy & $14(9-20)$ \\
\hline Median maximum radiation dose, (range), Gy & $33(14-50)$ \\
\hline Median prescription isodose, (range), $\%$ & $40(30-71)$ \\
\hline
\end{tabular}

Abbreviations: FU, follow up; GKRS, gamma knife radiosurgery.

In univariate analysis, factors associated with shorter PFS included parasellar invasion $(p=0.034)$ and tumor margin dose $(<13$ Gy) $(p=0.004)$. In multivariate analysis, only tumor margin dose was significantly associated with tumor progression (hazard ratio $[\mathrm{HR}]=3.526,95 \%$ confidence interval $[\mathrm{CI}]=1.400-8.877, \mathrm{p}=0.007)$ (Table 3). Kaplan-Meier plot generated for PFS (tumor margin dose <13 Gy VS tumor margin dose $\geq 13 \mathrm{~Gy}$ ) was shown in Figure 3.

\section{Hormonal outcomes}

The median endocrine follow-up was 60.6 months (Range: 14.5-169.4.0 months). Of the 80 patients with complete endocrine data before and after GKRS, 51 patients (63.8\%) presented with hypopituitarism before GKRS, including hypogonadism $(n=48)$, hypothyroidism $(n=15)$ and hypocortisolism $(n=11)$. Of the 29 patients $(36.3 \%)$ 
with normal endocrine function before GKRS, 7 patients $(24.1 \%)$ developed completely new hypopituitarism, including hypogonadism $(n=5)$, hypothyroidism $(n=4)$ and hypocortisolism $(n=3)$. The median time for completely new hypopituitarism in these patients was 37.56 months (Range: 16.2-66.8 months). Finally, of the 80 patients, 22 patients $(27.5 \%)$ developed new hypopituitarism, including hypogonadism $(n=7)$, hypothyroidism $(n=9)$, hypocortisolism $(n=15)$ and GH deficiency $(n=1)$ (Table 2). The median time until detection of new hypopituitarism after GKRS was 36.5 months (Range: 9.4 - 110.6 months). The cumulative rates of developing new hypopituitarism at 1, 3, 5 and 10 years was $4 \%, 21 \%, 30 \%$ and $57 \%$, respectively (Figure $4)$.
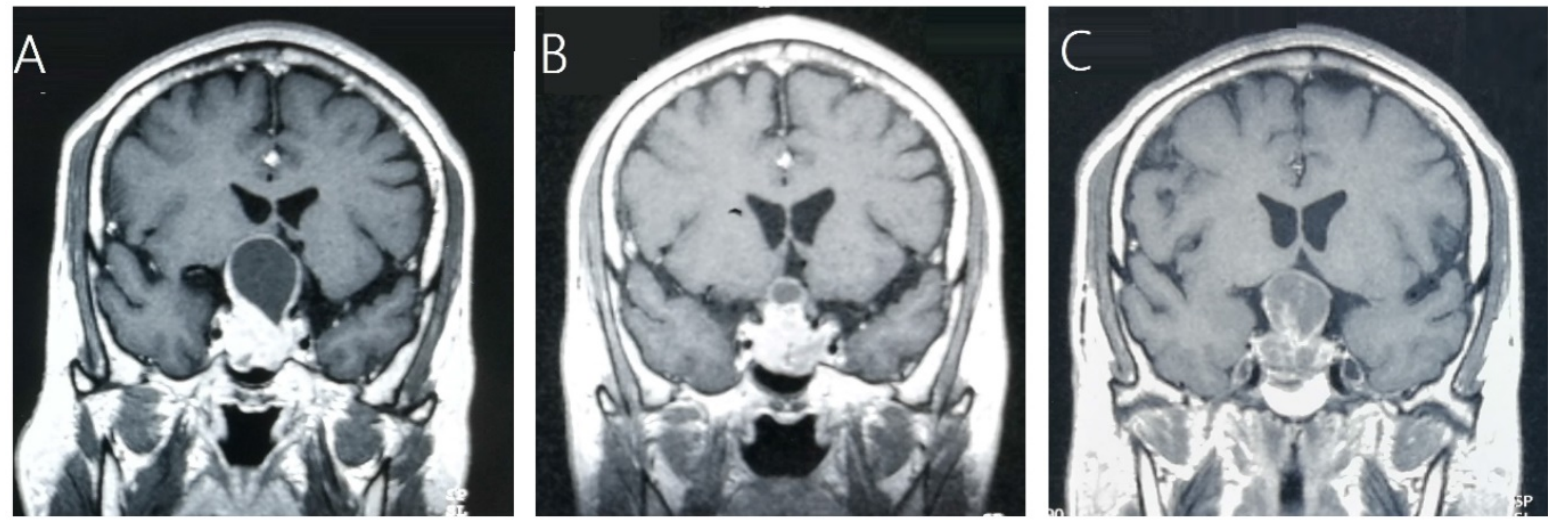

Figure 1. A 45-year-old male patient with residual NFPA received GKRS (10.5Gy/30\%) at 4.9 months after surgery and tumor progression due to progressive cystic enlargement was detected at 32.5 months after GKRS. A, MRI showed pituitary giant adenoma in sellar area. B, MRI showed residual tumor at 4.9 months after surgery. C, MRI showed tumor enlargement due to progressive cystic enlargement at 32.5 months after GKRS

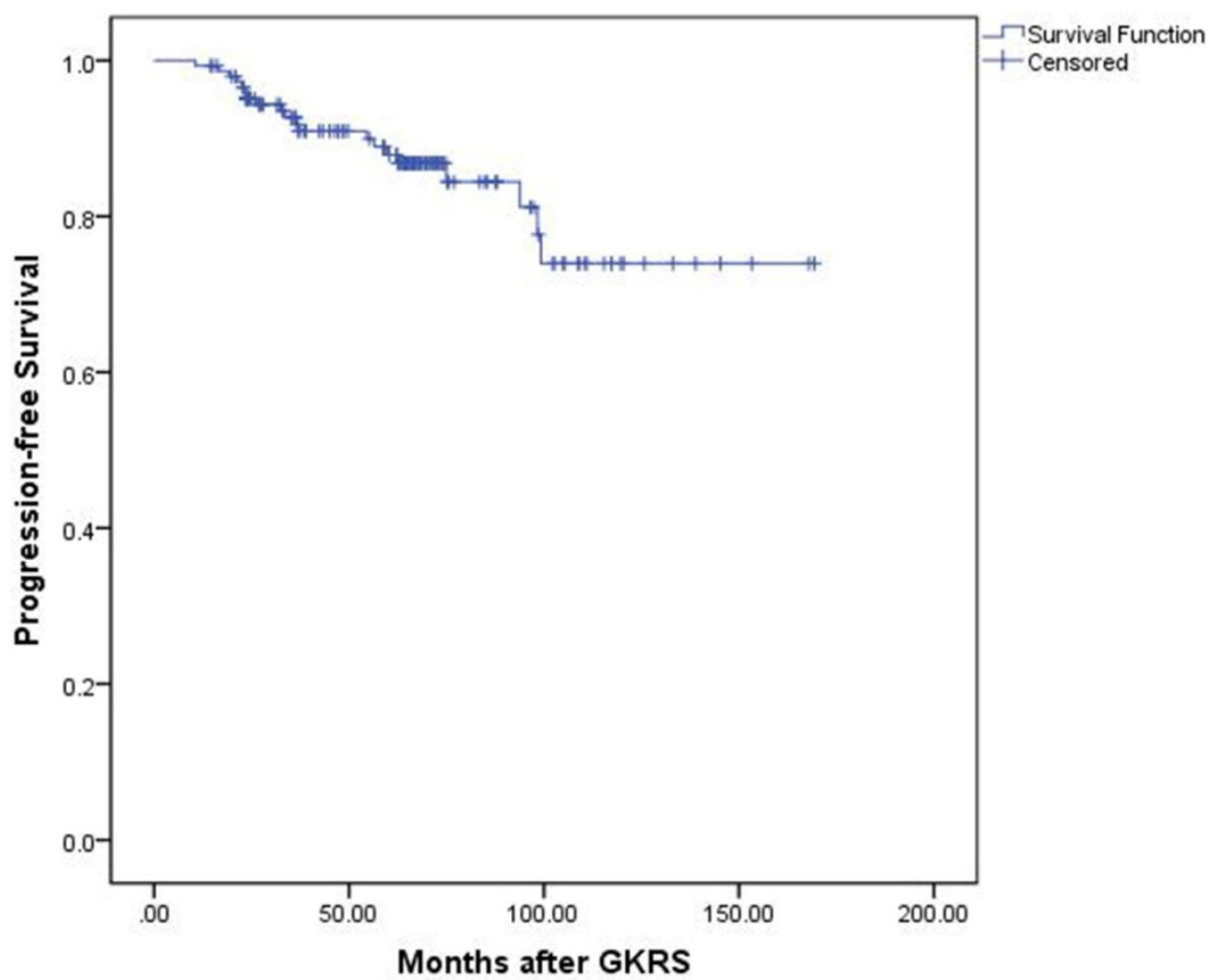

Figure 2. Kaplan-Meier curve of progression-free survival for the entire series. 
Table 3. Results of univariate and multivariate analysis for tumor control and new hypopituitarism after GKRS

\begin{tabular}{|c|c|c|c|c|c|}
\hline \multirow[t]{2}{*}{ Variables } & \multicolumn{4}{|l|}{ Tumor control } & \multirow{2}{*}{$\begin{array}{l}\text { New hypopituitarism } \\
\text { Univariate, } \mathrm{p}\end{array}$} \\
\hline & Univariate, $p$ & Multivariate, $p$ & HR & $95 \%$ CI & \\
\hline Age ( $\geq 55$ years) & 0.534 & NA & NA & NA & 0.721 \\
\hline Sex (male VS female) & 0.299 & NA & NA & NA & 0.849 \\
\hline Parasellar invasion & $0.034^{*}$ & 0.082 & NA & NA & 0.903 \\
\hline Suprasellar extension & 0.806 & NA & NA & NA & 0.695 \\
\hline Tumor margin dose (<13 Gy) & $0.004^{*}$ & $0.007^{*}$ & 3.526 & $1.400-8.877$ & 0.529 \\
\hline Tumor volume $\left(\geq 4 \mathrm{~cm}^{3}\right)$ & 0.255 & 0.932 & NA & NA & 0.545 \\
\hline Tumor type (recurrent VS residual) & 0.695 & NA & NA & NA & 0.963 \\
\hline Tumor response & NA & NA & NA & NA & 0.069 \\
\hline
\end{tabular}

Abbreviations: GKRS, gamma knife radiosurgery; CI, confidential interval; HR, hazards ratio; NA, not available.

*Statistically significant $(\mathrm{P}<0.05)$.

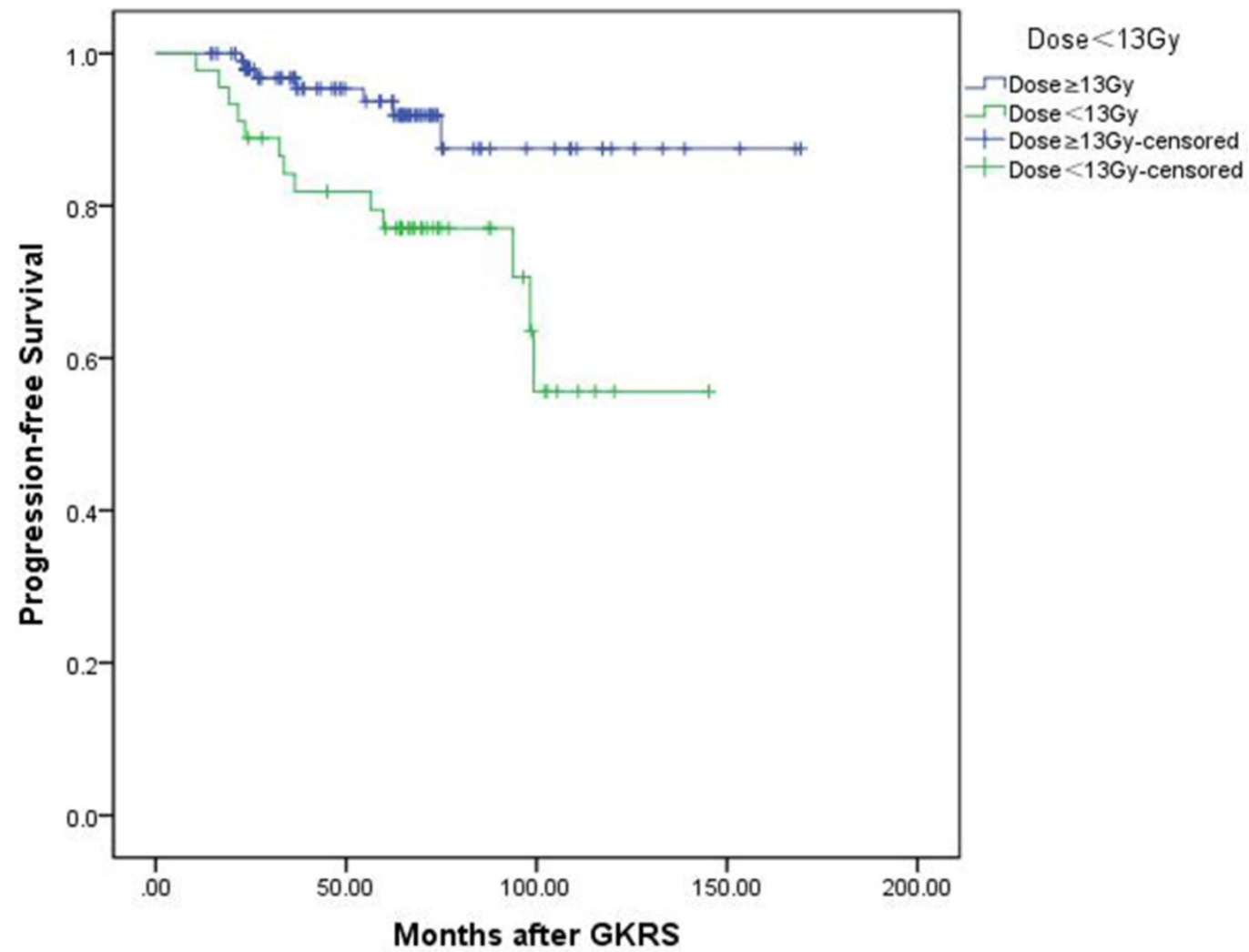

Figure 3. Kaplan-Meier curve of progression-free survival of tumor margin dose $<13$ Gy VS $\geq 13$ Gy. Low tumor margin dose (<13 Gy) shows shorter progression-free survival. $(p=0.004)$.

In univariate and multivariate analysis, there were no factors significantly associated with new hypopituitarism (Table 3).

\section{Clinical outcomes}

After GKRS, clinical outcomes, including visual and cranial nerve function, were evaluated. Six patients $(4.1 \%)$ worsened in visual function due to compression of chiasm by tumor progression after GKRS. However, visual dysfunction may also be influenced by nature of the visual dysfunction and GKRS. Finally, 4 patients $(2.7 \%)$ suffered new cranial dysfunction after GKRS (two patients with $\mathrm{CN} \mathrm{V}$ dysfunction, one patient with CN VI dysfunction, another patient CN III, IV, and VI dysfunction). All 4 patients had large tumors with parasellar invasion. Tumor shrinkage was observed in three patients and of these patients with $\mathrm{CN}$ dysfunction, these may be caused by GKRS. Tumor progression and invasion into the cavernous sinus and retrobulbar space subsequently caused CN III, IV, and VI dysfunction in another patient (Table 2).

\section{Discussion}

NFPAs are often detected due to symptoms and clinical signs. Transsphenoidal surgical resection with minimal complications is the recommended first-line treatment for most symptomatic patients. It has the advantages of rapid decompression of surrounding structures and pathological analysis. However, for 
tumors with suprasellar extension and parasellar invasion, complete surgical resection may be difficult to achieve (46\%-75\% of all surgical patients) $[6,17,18]$. The incidence of recurrent tumor after gross total resection occurred in $10 \%-20 \%$ of cases [2-6]. For patients following subtotal resection without adjuvant therapy, the rate of tumor progression ranged from $50 \%$ to $60 \%$ [2-5]. In a series of 126 postsurgical residual NFPA patients, Gittoe et al [19] reported progression free survival rates of $93 \%$ at 5,10 and 15 years for patients with residual NFPAs treated with RT after surgery, and $68 \%, 47 \%$ and $33 \%$ for those patients with observation, respectively. Other studies $[2,20]$ also reported similar results. Given the high rate of recurrent or residual NFPAs after surgical resection and tumor progression after surgery without radiotherapy, further management is generally required.

\section{Radiation techniques for postsurgical residual or recurrent NFPAs}

Radiation techniques have developed from 3D conformal radiotherapy (3D-CRT) to stereotactic radiosurgery (SRS) or fractionated stereotactic radiotherapy (FSRT). In previous studies of postsurgical conventional radiotherapy for NFPAs, tumor control rates were $80-90 \%$ at 10 years and $75-90 \%$ at 20 years with doses ranging from 45 to 55 Gy at 1.8-2.0 Gy per fraction [21]. The hypopituitarism occurred in $20 \%$ to $30 \%$ at 5 years after conventional radiotherapy[21]. SRS or FSRT which has advantages of precise tumor localization and a better dose conformity than conventional radiotherapy is the most commonly used radiation techniques and essential part in the treatment of pituitary adenomas. As GKRS is still considered the gold standard of SRS, GKRS is the most reported in literatures. For FSRT, tumor control rate was around $95 \%$ at 5 years and new hypopituitarism was reported in $10 \%$ to $48 \%$ in NFPA patients with doses ranging from 45-54 Gy at 1.8-2.0 Gy per fraction [21]. GKRS has been proved to offer a high tumor control rate of $83 \%-92 \%$ and a low rate of $9 \%-32 \%$ of new hypopituitarism for pituitary adenomas $[8,9,11,14,22,23]$. Tumor local control and complications were similar between GKRS and FSRT. However, the superiority of GKRS over FSRT for NFPA patients according to tumor control and complications has yet to be defined.

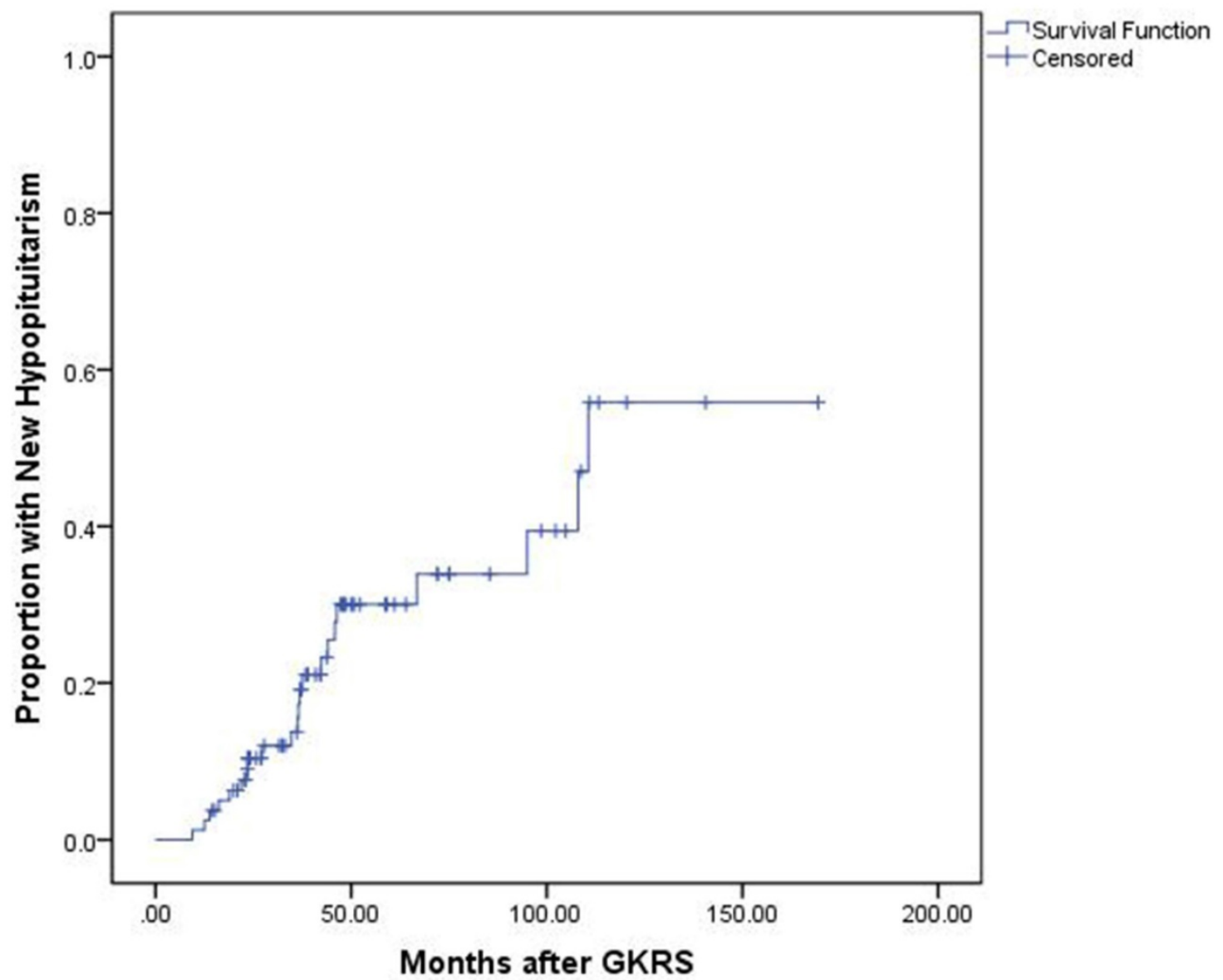

Figure 4. Kaplan-Meier curve of proportion with new hypopituitarism for the entire series. 


\section{Timing of radiosurgery}

The effect of timing of radiosurgery for residual or recurrent NFPAs had been discussed in previous reports. Pomeraniec et al. [24] conducted a multicenter retrospective study to evaluate the effect of timing of radiosurgery on outcomes. In this study, early GKRS ( $\leq 6$ months after resection) was related with a lower risk of radiological progression compared with delayed radiosurgery ( $>6$ months after resection). What's more, the rate of delayed endocrinopathy was not related to the timing of radiosurgery. In the study of Sadik et al. [25], adjuvant radiosurgery ( $\leq 6$ months after surgery) also provided similar tumor control rate with delayed GKRS, but there was a trend that adjuvant GKRS may provide a fewer endocrinologic deficits. It seemed that early GKRS should be used for residual NFPAs after surgery. In the current study, the timing of GKRS ( $\leq 6$ months after surgery versus $>6$ months after surgery) was not associated with tumor progression $(p=0.516)$ and new hypopituitarism $(\mathrm{p}=0.336)$. Tumor characteristic (residual VS recurrent) was not related with tumor progression $(\mathrm{p}=0.695)$ and new hypopituitarism $(p=0.963)$. As a delayed GKRS for postsurgical residual NFPAs would increase the risk of tumor regrowth and visual dysfunction, we suggested early GKRS should be used after surgical resection.

\section{Tumor control and related risk factors with GKRS}

Adjuvant GKRS has been proved to offer a high tumor control rate of $83 \%-92 \%$ for pituitary adenomas, as well as a low risk of complications. Gopalan et al. [14] reported a tumor control rate of $83 \%$ in 45 NFPA patients after GKRS. Lee et al. [22] reported 41 NFPA patients underwent GKRS as primary management. The overall tumor control rate was $92.7 \%$, and the tumor control rate was $94 \%$ and $85 \%$ at 5 and 10 years postradiosurgery, respectively. In the current study, 148 cases of postsurgical residual or recurrent NFPA patients underwent GKRS. The median tumor margin dose was 14 Gy (Range: 9-20 Gy). The progression-free survival rates were $99 \%$, $91 \%, 88 \%$ and $74 \%$ at $1,3,5$ and 10 years after postsurgical GKRS, respectively, which was similar with other studies.

There are many potential risk factors associated with tumor control, such as tumor margin dose, tumor volume, parasellar invasion and suprasellar extension. Gopalan et al. [14] reported that tumor volumes greater than $5 \mathrm{ml}$ at the time of GKRS were significantly associated with tumor growth $(p=0.003)$ based on 48 NFPA patients treated with GKRS. They also noted tumor margin doses less than 12 Gy was significantly associated with a lower tumor control rate $(33 \%)$ relative to doses of 12 Gy or higher $(80 \%)$. In the study of Park et al. [26], lower marginal dose $(<14$ Gy), larger tumor volume $(\geq 4.5 \mathrm{ml})$, and $\geq 2$ prior recurrences were associated with shorter PFS in univariate analysis based on 125 NFPA patients undergoing GKRS; larger $(\geq 4.5 \mathrm{ml})$ tumor volume $(p$ $=0.04 ; \mathrm{HR}=5.413)$, and $\geq 2$ prior recurrences $(\mathrm{p}=0.01$; $\mathrm{HR}=5.777)$ were related with shorter PFS in multivariate analysis. Sun et al. [8] reported 204 cases of residual or recurrent NFPA patients treated with GKRS. In the study, parasellar invasion affected tumor control significantly after adjusting for all other covariates $(\mathrm{HR}=3.705,95 \% \mathrm{CI}=1.248-11.000, \mathrm{p}=0.018)$. In our study, parasellar invasion and tumor margin dose were related with tumor control in univariate analysis. Only tumor margin dose was independently related with tumor control (HR=3.526, 95\% $\mathrm{CI}=1.400-8.877, \mathrm{p}=0.007)$ in multivariate analysis. There were 140 patients $(94.6 \%)$ with suprasellar extension, and 77 patients (52\%) with parasellar invasion in this study. The average tumor volume was $6.5 \mathrm{ml}$. Large tumor volume, parasellar and suprasellar extension were risk factors for tumor progression. A relative low tumor margin dose $(<13$ Gy) was not enough for tumor control in those patients with so many risk factors. For residual or recurrent NFPA patients with large tumor volume, repeat surgery should be considered to reduce tumor volume as much as possible.

\section{New hypopituitarism after GKRS and related risk factors}

Hypopituitarism is the most common complication after GKRS for pituitary adenoma patients. Previous reports showed the rate of new hypopituitarism at almost 9\%-32\% [8-13, 23]. Risk factors such as tumor volume, tumor margin dose, higher pituitary gland dose and history of radiation, were potentially related with new hypopituitarism. Pollock et al. [13] reported the rate of new hypopituitarism was $18 \%$ and $50 \%$ at 5 years after SRS for tumors volume $\leq 4 \mathrm{~cm}^{3}$ and $>4 \mathrm{~cm}^{3}$ respectively. In the study of Lee et al. [22], tumor margin dose $>18$ Gy and a maximum dose $>36$ Gy were independent prognostic factors related with hypopituitarism. Graffeo et al. [27] found a lower rate of hypopituitarism in patients with a mean gland dose $<11$ Gy. The rate of hypopituitarism at 2-years and 5 -years were $2 \%$ and $5 \%$ for a mean gland dose $<11$ Gy, whereas the rate of hypopituitarism was $31 \%$ and $51 \%$ at 2-years and 5-years for patients with a mean gland dose $\geq 11$ Gy. A correlation was also found between prior radiotherapy and new hypopituitarism 
after SRS in the study of Park et al. [26]. However, in our study, no factors were significantly correlated with new hypopituitarism and the rate of new hypopituitarism was similar to other studies. From what was discussed above, compression by tumor mass and radiation dose exposition to the normal pituitary gland were the main causes of new hypopituitarism after GKRS in patients with NFPA. Hence, decompression of pituitary gland by primary total or subtotal surgical resection and dose reduction in pituitary gland without sacrificing radiation dose to tumor may be contributed to reduce the rate of new hypopituitarism after GKRS for patients with pituitary adenomas.

\section{Other complications with GKRS}

The incidence of neurological complications such as visual impairment and cranial nerve dysfunction were relatively low after GKRS, which was reported to be between $0 \%$ and $5 \%[11,23,26]$. In this study, six patients $(4.1 \%)$ developed new or worsening visual dysfunction. Four patients $(2.7 \%)$ developed new or worsening cranial neuropathy. Visual dysfunction and cranial nerve dysfunction within the cavernous sinus could be caused by compression and radiation damage, especially in patients with suprasellar invasion and parasellar extension. The optic pathway was generally restricted to $<8$ Gy in GKRS [28]. That may sacrifice radiation dose to the tumor near the optic pathway. The dose of up to 40 Gy appears to be a relatively safe to the cranial nerves of the cavernous sinus [28].

\section{Study limitations}

In this study, some limitations should be noted. First, this was a retrospective study and therefore contained information and selection biases. Second, taking into account the irregular shape of postsurgical residual or recurrent pituitary adenomas, tumor volume measurement was only a rough estimate of the actual volume. Third, as some patients were from long distances and had poor compliance, many patients did not routinely follow up with endocrine evaluations; only 80 patients (54.1\%) with complete endocrine evaluations before and after GKRS were analyzed for new hypopituitarism, the small number of cases may limit statistical power. Four, although all patients were histologically confirmed as NFPAs, there was a lack of pathological markers of aggressive behavior, which may be associated with tumor regrowth. Finally, given the lack of IGF-1 or a stimulation test in some patients followed up in rural area, GH deficiency may be under estimated.

\section{Conclusion}

Surgical resection is the recommended first-line treatment for NFPA patients. For residual and recurrent NFPA patients after surgery, GKRS has been used for decades. In this study, GKRS can offer a high tumor control rate as well as a low rate of complications in postsurgical residual or recurrent NFPA patients.

\section{Acknowledgements}

\section{Author contributions}

Research idea and study design: Yinhui Deng, $\mathrm{Xi}$ Li, Jinxiu Yu.

Data acquisition: Yinhui Deng, Yanli Li, Xi Li, Tingting Quan, Chao Peng, Junyi Fu, Xin Yang, Lisha $\mathrm{Wu}$, Jinxiu $\mathrm{Yu}$.

Statistical analysis: Yanli Li, Xi Li, Lisha Wu.

Manuscript drafting: Yanli Li, Yinhui Deng, Xi

Li.

Supervision and mentorship: Jinxiu Yu.

Each author contributed important content during manuscript drafting or revision and accepts accountability for the overall work, and all the authors agreed on the final manuscript.

\section{Funding}

This work was supported by National Key Research and Development Project (grants number: 2017YFC0113700); National Natural Science Foundation of China (grants number: 81800682); the Medical Science and Technology Research Fund Project of Guangdong (grants number: A2018443); the Medical and Health Project of Guangdong (grants number: 20181A011069).

\section{Competing Interests}

The authors have declared that no competing interest exists.

\section{References}

1. Hoybye C, Rahn T. Adjuvant Gamma Knife radiosurgery in non-functioning pituitary adenomas; low risk of long-term complications in selected patients. Pituitary. 2009; 12: 211-6.

2. Park P, Chandler WF, Barkan AL, Orrego JJ, Cowan JA, Griffith KA, et al. The role of radiation therapy after surgical resection of nonfunctional pituitary macroadenomas. Neurosurgery. 2004; 55: 100-6; discussion 6-7.

3. Picozzi P, Losa M, Mortini P, Valle MA, Franzin A, Attuati L, et al. Radiosurgery and the prevention of regrowth of incompletely removed nonfunctioning pituitary adenomas. Journal of neurosurgery. 2005; $102 \mathrm{Suppl}$ : 71-4.

4. Ferrante E, Ferraroni M, Castrignano T, Menicatti L, Anagni M, Reimondo G, et al. Non-functioning pituitary adenoma database: a useful resource to improve the clinical management of pituitary tumors. European journal of endocrinology. 2006; 155: 823-9.

5. Dallapiazza RF, Grober Y, Starke RM, Laws ER, Jr., Jane JA, Jr. Long-term results of endonasal endoscopic transsphenoidal resection of nonfunctioning pituitary macroadenomas. Neurosurgery. 2015; 76: 42-52; discussion -3.

6. Chang EF, Zada G, Kim S, Lamborn KR, Quinones-Hinojosa A, Tyrrell JB, et al. Long-term recurrence and mortality after surgery and adjuvant radiotherapy for nonfunctional pituitary adenomas. Journal of neurosurgery. 2008; 108: 736-45. 
7. Sheehan JP, Starke RM, Mathieu D, Young B, Sneed PK, Chiang VL, et al. Gamma Knife radiosurgery for the management of nonfunctioning pituitary adenomas: a multicenter study. Journal of neurosurgery. 2013; 119: 446-56.

8. Sun S, Liu A, Zhang Y. Long-Term Follow-Up Studies of Gamma Knife Radiosurgery for Postsurgical Nonfunctioning Pituitary Adenomas. World neurosurgery. 2019.

9. Bir SC, Murray RD, Ambekar S, Bollam P, Nanda A. Clinical and Radiologic Outcome of Gamma Knife Radiosurgery on Nonfunctioning Pituitary Adenomas. Journal of neurological surgery Part B, Skull base. 2015; 76: 351-7.

10. Starke RM, Williams BJ, Jane JA, Jr., Sheehan JP. Gamma Knife surgery for patients with nonfunctioning pituitary macroadenomas: predictors of tumor control, neurological deficits, and hypopituitarism. Journal of neurosurgery. 2012; 117: 129-35.

11. Losa M, Valle M, Mortini P, Franzin A, da Passano CF, Cenzato M, et al. Gamma knife surgery for treatment of residual nonfunctioning pituitary adenomas after surgical debulking. Journal of neurosurgery. 2004; 100: 438-44.

12. Zibar Tomsic K, Dusek T, Kraljevic I, Heinrich Z, Solak M, Vucinovic A, et al. Hypopituitarism after gamma knife radiosurgery for pituitary adenoma. Endocrine research. 2017; 42: 318-24.

13. Pollock BE, Cochran J, Natt N, Brown PD, Erickson D, Link MJ, et al. Gamma knife radiosurgery for patients with nonfunctioning pituitary adenomas: results from a 15-year experience. International journal of radiation oncology, biology, physics. 2008; 70: 1325-9.

14. Gopalan R, Schlesinger D, Vance ML, Laws E, Sheehan J. Long-term outcomes after Gamma Knife radiosurgery for patients with a nonfunctioning pituitary adenoma. Neurosurgery. 2011; 69: 284-93.

15. Yu J, Li Y, Quan T, Li X, Peng C, Zeng J, et al. Initial Gamma Knife radiosurgery for nonfunctioning pituitary adenomas: results from a 26-year experience. Endocrine. 2020.

16. Mak HK, Lai SW, Qian W, Xu S, Tong E, Vance ML, et al. Effective time window in reducing pituitary adenoma size by gamma knife radiosurgery. Pituitary. 2015; 18: 509-17.

17. Mortini P, Losa M, Barzaghi R, Boari N, Giovanelli M. Results of transsphenoidal surgery in a large series of patients with pituitary adenoma. Neurosurgery. 2005; 56: 1222-33; discussion 33

18. Kurosaki M, Ludecke DK, Flitsch J, Saeger W. Surgical treatment of clinically nonsecreting pituitary adenomas in elderly patients. Neurosurgery. 2000; 47: 843-8; discussion 8-9.

19. Gittoes NJ. Radiotherapy for non-functioning pituitary tumors--when and under what circumstances? Pituitary. 2003; 6: 103-8

20. Woollons AC, Hunn MK, Rajapakse YR, Toomath R, Hamilton DA, Conaglen $\mathrm{JV}$, et al. Non-functioning pituitary adenomas: indications for postoperative radiotherapy. Clinical endocrinology. 2000; 53: 713-7.

21. Minniti G, Flickinger J, Tolu B, Paolini S. Management of nonfunctioning pituitary tumors: radiotherapy. Pituitary. 2018; 21: 154-61.

22. Lee CC, Kano H, Yang HC, Xu Z, Yen CP, Chung WY, et al. Initial Gamma Knife radiosurgery for nonfunctioning pituitary adenomas. Journal of neurosurgery. 2014; 120: 647-54

23. Sheehan JP, Kondziolka D, Flickinger J, Lunsford LD. Radiosurgery for residual or recurrent nonfunctioning pituitary adenoma. Journal of neurosurgery. 2002; 97: 408-14.

24. Pomeraniec IJ, Kano H, Xu Z, Nguyen B, Siddiqui ZA, Silva D, et al. Early versus late Gamma Knife radiosurgery following transsphenoidal surgery for nonfunctioning pituitary macroadenomas: a multicenter matched-cohort study. Journal of neurosurgery. 2018; 129: 648-57.

25. Sadik ZHA, Voormolen EHJ, Depauw P, Burhani B, Nieuwlaat WA, Verheul J, et al. Treatment of Nonfunctional Pituitary Adenoma Postoperative Remnants: Adjuvant or Delayed Gamma Knife Radiosurgery? World neurosurgery. 2017; 100: 361-8.

26. Park KJ, Kano H, Parry PV, Niranjan A, Flickinger JC, Lunsford LD, et al. Long-term outcomes after gamma knife stereotactic radiosurgery for nonfunctional pituitary adenomas. Neurosurgery. 2011; 69: 1188-99.

27. Graffeo CS, Link MJ, Brown PD, Young WF, Jr., Pollock BE. Hypopituitarism After Single-Fraction Pituitary Adenoma Radiosurgery: Dosimetric Analysis Based on Patients Treated Using Contemporary Techniques. International journal of radiation oncology, biology, physics. 2018; 101: 618-23.

28. Tishler RB, Loeffler JS, Lunsford LD, Duma C, Alexander E, 3rd, Kooy HM, et al. Tolerance of cranial nerves of the cavernous sinus to radiosurgery. International journal of radiation oncology, biology, physics. 1993; 27: 215-21. 